\title{
Intravenous paracetamol vs intranasal desmopressin for renal colic in the emergency department: A randomized clinical trial
}

Ghafouri H.B ${ }^{\mathrm{a}}$, Abazarian $\mathrm{N}^{\mathrm{b}}$, Yasinzadeh $\mathrm{M}^{\mathrm{a}}$, Modirian Ehsan ${ }^{\mathrm{c}}$

${ }^{\text {a }}$ Emergency Medicine, Trauma and Injury Research Center, Iran University of Medical Sciences, Tehran, Iran

${ }^{\mathrm{b}}$ Emergency Medicine, Alborz University of Medical Sciences, Karaj, Iran

${ }^{c}$ Emergency Medicine, Qazvin University of Medical Sciences, Qazvin, Iran

\section{Abstract}

Objective. To evaluate the analgesic efficacy of intranasal desmopressin alone vs intravenous paracetamol in patients referred to the emergency department with renal colic. Design. Randomized clinical trial. Setting. This study was conducted in the emergency unit of a university hospital. Subjects. Patients referred to the emergency room with renal colic. Primary Outcome. Effect of intranasal desmopressin in pain relief in comparison with intravenous paracetamol. Methods. In this trial, 240 patients diagnosed with renal colic were randomly divided into two groups to compare the analgesic effect of intravenous paracetamol $(15 \mathrm{mg} / \mathrm{kg})$ and intranasal desmopressin spray $(40 \mu \mathrm{g})$. Pain scores were measured by a numeric rating scale at baseline and after 15, 30, and 60 minutes. Adverse effects and need for rescue analgesic $(0.05 \mathrm{mg} / \mathrm{kg} \mathrm{max} 3 \mathrm{mg}$ morphine sulphate) were also recorded at the end of the study. Results. Three hundred patients were eligible for the study; however, 240 were included in the final analysis. The patients in the two groups were similar in their baseline characteristics and baseline pain scores. The mean pain score after 15 minutes was more reduced and was clinically significant $(>3)$ in the desmopressin group $(\mathrm{P}<0.0001)$. There was no significant difference between mean pain scores in the two groups after 30 minutes $(\mathrm{P}=0.350)$ or 60 minutes $(\mathrm{P}=0.269)$, but the efficacy of the two drugs was significant in terms of pain reduction $(>6)$. Conclusions. Our study showed that intranasal desmopressin is as effective as intravenous paracetamol for renal colic pain management; however, significant clinical reduction in pain score occurred faster with intranasal desmopressin.

Keywords: Desmopressin; Emergency; Hospital; Pain; Paracetamol; Renal colic 